\title{
Antepartum Evaluation of the Fetus and Fetal Well Being
}

\author{
ERICA O'NEILL, MD and JOHN THORP, MD \\ Department of Obstetrics and Gynecology, University of North Carolina, Chapel Hill, North \\ Carolina
}

\begin{abstract}
Despite widespread use of many methods of antenatal testing, limited evidence exists to demonstrate effectiveness at improving perinatal outcomes. An exception is the use of Doppler ultrasound in monitoring high-risk pregnancies thought to be at risk of placental insufficiency. Otherwise, obstetricians should proceed with caution and approach the initiation of a testing protocol by obtaining an informed consent. When confronted with an abnormal test, clinicians should evaluate with a second antenatal test and consider administering betamethasone, performing amniocentesis to assess lung maturity, and/or repeating testing to minimize the chance of iatrogenic prematurity in case of a healthy fetus.
\end{abstract}

\section{Keywords}

antenatal testing; fetal well-being; fetal movement counting; nonstress test; fetal lung maturity; Doppler ultrasound

\section{Introduction}

The primary goal of antenatal evaluation is to identify fetuses at risk for intrauterine injury and death so that intervention and timely delivery can prevent progression to stillbirth. Ideally, antenatal tests would decrease fetal death without putting large numbers of healthy fetuses at risk for premature delivery and the associated morbidity and mortality. Despite widespread use of many tests, limited evidence exists to demonstrate effectiveness at improving perinatal outcomes with application of these tests.

Certainly, there is a definite need to find a reliable test of fetal well-being. The National Center for Health Statistics defines perinatal mortality rate (PMR) as the number of late fetal deaths (28-wk gestation or more) plus early neonatal deaths (infants 0 to $6 \mathrm{~d}$ of age) per 1000 live births plus fetal deaths. In the United States, stillbirths account for more than 55\% of the perinatal mortality ${ }^{1}$ and could potentially be prevented with an effective form of fetal testing.

Unfortunately, many barriers need to be overcome in the development of a reliable assessment of fetal well-being. First, the basis of fetal testing rests on the hypothesis that a compromised fetus undergoes a series of detectable physiological changes such as the redistribution of blood flow or decreasing unnecessary movements. However, in the third trimester, normal fetuses spend $25 \%$ of their time in a quiet sleep state. During the normal 
sleep state the fetal heart rate variability is reduced, and there may be infrequent breathing movements. ${ }^{2}$ Tests performed while a fetus is in a resting state may be incorrectly classified as an abnormal test. Therefore, when using tests to evaluate fetal condition, clinicians need to appreciate whether a fetus is simply in a temporary quiet sleep state or neurologically compromised.

Second, which pregnancies may benefit from testing is unclear. Observational studies show an increased PMR in high-risk pregnancies, with a 10-fold difference in perinatal mortality between high-risk and low-risk groups (70/1000 vs. 7/1000). ${ }^{3}$ Yet the overall prevalence of these high-risk conditions is low and 30\% to $50 \%$ of perinatal deaths actually occur in lowrisk patients. Therefore if a sensitive and specific test existed, all women would benefit from some form of fetal testing. Targeting only high-risk women would still be inadequate.

Third, the prevalence of an abnormal condition has the greatest impact on the predictive capability of an antepartum test. Although stillbirths are devastating, they are unlikely even among high-risk women and very rare among low-risk women. In many cases an abnormal test may be more likely to indicate a false positive (healthy fetus), instead of a true positive (compromised fetus). ${ }^{2}$ Even in the case of high-risk pregnancies with a prevalence of $70 / 1000$, a test that is $99 \%$ sensitive and specific only has a positive predictive value of $88 \%$.

Finally, to be successful at preventing death, an antepartum test needs to identify a compromised fetus in enough advanced time for an intervention to be successful. No known method of assessment can predict sudden events, such as a cord accident or placental abruption, which are frequent causes of fetal death. It is important for the clinician to keep these limitations in mind when evaluating the merits of an antenatal test of fetal well-being.

\section{Maternal Assessment of Fetal Activity}

Fetal movement counting is an evaluation method which pregnant women quantify the fetal movements they feel. In theory, decreased movement alerts the mother of a deteriorating fetal condition. She can then bring this to the attention of health care providers who can evaluate further and intervene as needed to prevent fetal death. Because it can be performed each day, or multiple times daily, it has advantages over other fetal tests that cannot practically be performed this frequently.

Women who report decreased fetal movement have an incidence of stillbirth that is 60 times higher than women without this complaint. ${ }^{2}$ Although decreased fetal movement commonly precedes fetal death, it does not necessarily indicate fetal death is imminent. Many factors other than worsening fetal condition can influence the perception of movement, including maternal activity, position, obesity, medications, gestational age, placental location, and amniotic fluid volume. Fetal movement also varies normally over the course of the day, peaking between $9_{\mathrm{PM}}$ and 1 Ам when maternal glucose levels are falling. ${ }^{2}$

The most commonly recommended counting technique is the count-to-ten method where the woman is instructed each day to count and record the time she feels the 10th fetal movement. Studies use a variety of definitions of the "alarm," the time when a woman should seek evaluation immediately if she has not felt 10 kicks. What constitutes a clinically important change in the maternal perception of fetal movement is unknown, although most experts currently recommend that women seek care after 2 hours. A later alarm has a low sensitivity to identify compromised fetuses, whereas an earlier alarm generates an unacceptably high false-positive rate (Table 1$).{ }^{4}$ Our inability to define an appropriate alarm for women is undermining fetal movement counting as a means to reduce adverse perinatal outcomes. 
A prospective study of fetal movement counting in low-risk women noted a fetal mortality rate of 8.7/1000 during a 7-month control period, which then decreased significantly to 2.1/1000 during the study time period. ${ }^{5}$ Patients used the count-to-ten approach with a 2hour alarm, and the number of antepartum tests performed to assess decreased fetal activity increased $13 \%$ during the study period. However, given that this was not a randomized trial it is impossible to identify the exact factor that contributed to the difference seen. This improvement could be due to some unmeasured difference in the 2 populations and not necessarily due to the fetal movement counting intervention.

The only randomized controlled trial evaluating formal fetal movement counting enrolled over 68,000 low-risk women and did not show a benefit. ${ }^{6}$ The antepartum death rate was equal in both groups, $2.9 / 1000$ in the intervention group versus 2.7/1000 in the control group. ${ }^{7}$ There were several problems with this study, including that the compliance for reporting decreased fetal movement was only $46 \%$ and that $9 \%$ of the control participants received formal fetal movement counting charts. These problems could have accounted for the lower than expected rate of fetal deaths in the control group and led to an internal bias that reduced the difference seen between the intervention and control groups. ${ }^{6}$ No randomized controlled trials have been performed looking at fetal movement counting specifically in high-risk women.

Not enough evidence is available to recommend or condemn formal fetal movement counting in either low-risk or high-risk women. The indirect evidence of the ability of formal fetal movement counting to identify fetuses at risk of intrauterine death was not translated into reduced perinatal mortality. ${ }^{6}$ The lack of high-quality randomized trials makes it difficult to draw definitive conclusions.

\section{Nonstress Test (NST)}

The cardiotocograph (CTG) is a continuous record of the fetal heart rate obtained by an ultrasound transducer placed on the mother's abdomen, with a second transducer placed over the uterine fundus to record uterine activity simultaneously. Components of the fetal heart rate that are assessed include baseline rate, variability, accelerations, and decelerations as well as the relationship between fetal heart rate and the timing of uterine contractions. ${ }^{8}$ CTG may be used in isolation, referred to as the "nonstress test," or with the stimulation of uterine contractions to see how the fetal heart responds, known as the "contraction stress test." The contraction stress test is time consuming to perform and requires an intravenous infusion of oxytocin in many cases. Contraindications limit use of the contraction stress test in many high-risk situations such as antepartum bleeding, placenta previa, preterm premature rupture of membranes, or a history of preterm labor. The contraction stress test suffers from many disadvantages, has shown minimal demonstrated benefits over other methods of fetal evaluation, and should not be used routinely. ${ }^{9}$

The nonstress test is used widely in current obstetrics practice. The false-negative rate for the nonstress test is low ranging from $0.19 \%$ to $1 \%$, and when assessing the interobserver variation the proportions of agreement for normal tests were high. ${ }^{10}$ In contrast, the falsepositive rate of a nonreactive nonstress test is as high as $55 \%,{ }^{11}$ and the proportions of agreement among experts for equivocal/ominous tests were poor. ${ }^{10}$ Given that with an abnormal test result a fetus is more likely to be healthy than compromised, results must be used cautiously by clinicians, particularly at early gestational ages when delivery could result in significant morbidity.

A meta-analysis of randomized controlled trials included 4 studies with a total of 1636 women. All women received antenatal CTG, but 1 group had the CTG results provided to clinicians managing their care, whereas the other group had the CTG results concealed. ${ }^{8}$ The 
4 studies only recruited women considered at increased risk of pregnancy complications. No significant difference was identified in the risk of perinatal mortality, which was $2.3 \%$ in the group with CTG results revealed versus $1.1 \%$ in the group with CTG results concealed. ${ }^{8}$ Looking at secondary outcomes, Apgar scores $<7$ at 5 minutes, admission to neonatal intensive care units, gestational age at birth, or neonatal seizures were not different between groups. All 4 studies were done in the 1980s, which may limit generaliz-ability to current practice. And even though the review includes all randomized trials, it is still underpowered to assess perinatal mortality. On the basis of these results, there is no benefit from nonstress test evaluation in isolation. However, fetal assessment in current practice often involves a combination of methods, which may reduce the relevance of the effectiveness of this single method of testing. ${ }^{8}$

\section{Biophysical Profile (BPP)}

The BPP is a composite test that collects 5 indicators of fetal well-being, including fetal heart rate reactivity, breathing movements, gross body movements, muscular tone, and quantitative estimation of amniotic fluid volume. ${ }^{3}$ The assessment of fetal heart rate is accomplished by performing a nonstress test, whereas the latter 4 variables are observed using real-time ultra-sonography. Two points are awarded for each parameter when present, and 0 when absent after 30 minutes of ultrasound observation. Equal weight is given to each of the 5 parameters. When each of the 4 ultrasound variables is normal, the non-stress test may be excluded as it adds little to the predictive accuracy of the BPP. ${ }^{12}$

The BPP is more time consuming and requires more technical skill to perform than the nonstress test. The false-negative rate of the BPP was calculated in a study with 19,221 high-risk pregnancies and was very low at $0.07 \% .{ }^{13}$ Unfortunately, the false-positive rate of the BPP is only slightly lower than with the nonstress test. ${ }^{11}$ Certainly, factors other than hypoxemia may affect BPP parameters including gestational age, administration of steroids, magnesium sulfate, labor, and excessive transducer pressure on maternal abdomen. ${ }^{12}$

A meta-analysis of randomized trials included 4 studies with a total of 2829 women. These studies compared the BPP with the nonstress test and 1 trial with 145 women comparing the BPP with the non-stress test combined with amniotic fluid assessment. ${ }^{12}$ Pregnancies were managed on the basis of normal or abnormal test results (without reference to the test performed) in 3 of the trials. When the BPP was compared with the nonstress test there was no significant difference between the groups in perinatal deaths in infants, Apgar score $<7$ at 5 minutes, and overall incidence of cesarean section. No randomized controlled trials have been performed comparing BPP with no fetal evaluation.

Available evidence from randomized trials does not support the use of the BPP as a test of fetal well-being in high-risk pregnancies. However, the studies included in the meta-analysis only evaluated a total of 2974 women, thus additional evidence is required to make any meaningful conclusions.

\section{Modified BPP}

Given that the BPP is more time consuming and requires more technical skill to perform, the modified BPP was developed. This test consists of a combination of a nonstress test as well as an ultrasound to estimate the amniotic fluid index (AFI). Typically further evaluation is performed when an abnormal result is obtained with the modified BPP.

A large prospective observational study used the modified BPP to evaluate 15,842 high-risk women. ${ }^{14}$ If the participant had a nonreactive NST or an abnormal AFI, a full BPP was performed. This is a commonly performed sequence in modern testing facilities in the 
United States. The false-negative rate of this antepartum testing protocol was 0.8 per 1000 women tested. However, the false-positive rate was high, $60 \%$ of those delivered because of an abnormal antepartum test had no evidence of fetal compromise, defined as intrapartum fetal distress, meconium-stained amniotic fluid, 5-minute Apgar scores $<7$, or birth weight $<10$ th percentile for gestational age. False-positive test results led to preterm delivery in $1.5 \%$ of those tested before term. ${ }^{14}$

The only randomized trial specifically looking at the modified BPP was small, and included in the meta-analysis discussed above for the BPP. It included 145 low-risk women with pregnancies after 42 weeks of gestation who were randomly allocated to fetal monitoring by either a full BPP using AFI or a modified BPP using maximum pool depth. Significantly more abnormal antenatal monitoring results occurred in the full BPP group ( $47.2 \%$ vs. $20.5 \%$ ). No differences were observed in cord blood gases, neonatal outcome, or in outcomes related to labor and delivery between the 2 groups, but a trend toward more obstetric interventions in the full BPP was noted. ${ }^{12}$

Overall, inadequate data exist to recommend or not recommend the modified BPP. However, the modified BPP requires less time and expertise to perform and has a similarly reassuring false-negative rate and potentially a lower false-positive rate than the nonstress test. Additional studies should be performed to evaluate its potential as a screening test.

\section{Fetal Doppler Ultrasound}

Fetal Doppler evaluation uses ultrasound to measure blood flow velocities in the fetal vessels, most commonly the umbilical artery. Flow is most often quantified either by pulsatility index or resistant index. These indices reflect the downstream vascular resistance by quantifying the differences between the peak systolic and the end-diastolic velocity within blood vessels of interest in each cardiac cycle. ${ }^{15}$ Ratios are created to allow the sonographer to not have to measure the angle of insonation, which is notoriously inaccurate. Normally growing fetuses have high-velocity diastolic flow. Growth-restricted fetuses often have diminished, absent, or even in severe cases reversed umbilical artery diastolic flow.

The odds ratio for perinatal mortality in growth-restricted pregnancies complicated by absent diastolic flow is 4.0. With reversed diastolic flow, the odds ratio for mortality is increased to 10.6. This increase in mortality with abnormal Doppler testing persists even after adjustment for gestational age. ${ }^{16}$ An absence of umbilical artery diastolic flow has been shown to be an accurate clinical test with a sensitivity of $78 \%$ for detecting hypoxia and a sensitivity of $90 \%$ for detecting acidosis on fetal blood gas at delivery. The positive predictive value of absent end-diastolic flow ranges from 53\% to $88 \%$ and negative predictive value is high at $98 \%$ to $100 \% .^{17}$

Of all the antenatal assessment methods, Doppler-based tests have been evaluated most rigorously in randomized trials. A meta-analysis combined 4 randomized trials and demonstrated that routine umbilical artery Doppler screening of all low-risk pregnancies does not reduce the risk of perinatal death. ${ }^{15}$ On the basis of a single study with 2016 lowrisk women, no significant difference was identified in serious neonatal morbidity, Apgar scores $<7$ at 5 minutes, cesarean section, operative vaginal births, induction of labor, or neonatal resuscitation. ${ }^{15}$

Doppler ultrasound may be effective in identifying at-risk fetuses specifically in high-risk pregnancies. In a meta-analysis of 18 randomized trials enrolling just over 10,000 high-risk women, the use of Doppler ultrasound to evaluate the fetal umbilical artery was associated with a significant reduction in perinatal deaths. ${ }^{18}$ The group that had Doppler results provided to the clinician had a perinatal death rate of $1.2 \%$ versus $1.7 \%$ in the group that 
clinicians did not have access to Doppler results. The number of infants needed to be assessed with Doppler to prevent a perinatal death was 203. In most of those studies Doppler ultrasound was used in addition to other fetal monitoring, such as NST or the BPP. However, 4 studies compared Doppler ultrasound alone with nonstress test alone in women whose pregnancies were considered at increased risk of problems. This analysis included 2813 women and had much less power for assessing the main clinical outcome. The perinatal mortality was not significantly different. ${ }^{18}$

Doppler studies can also be performed on other fetal vessels. Recently, considerable interest has been generated by observations that ductus venosus flow may be a good predictor of perinatal outcome. However, the effectiveness of this assessment has yet to be tested in large clinical trials. ${ }^{18}$

Doppler studies of the umbilical artery should be included in the protocols for fetal monitoring in high-risk pregnancies thought to be at risk of placental insufficiency. The clear definition of suspected placental insufficiency, frequency of Doppler studies, and timing of delivery in the presence of abnormal Doppler studies have not been determined. ${ }^{18}$

\section{Assessment of Fetal Lung Maturity}

Given the high false-positive rate of many antenatal tests, assessing the likelihood of respiratory distress syndrome (RDS) for the infant after premature delivery is beneficial. When contemplating early delivery, the risk of iatrogenic prematurity can be balanced against the risk of continued antepartum assessment of the potentially compromised fetus and can help determine ultimate management. ${ }^{2}$ RDS is caused by a deficiency of pulmonary surfactant. Tests of pulmonary maturity use different techniques to quantify the amount of surfactant present. With the exception of vaginal pool specimens, the evaluation of pulmonary maturity requires an amniocentesis to obtain a sample of amniotic fluid. A review of amniocentesis for assessment of fetal lung maturity in 913 patients showed a very low complication rate for this procedure. Only $1.6 \%$ of the amniocentesis procedures were unsuccessful and $0.7 \%$ required same-day delivery. ${ }^{2}$

The lecithin/sphingomyelin (L/S) ratio was the first reliable assay. A ratio of 2.0 or greater has repeatedly been associated with pulmonary maturity. A mature L/S ratio predicts the absence of RDS in $98 \%$ of neonates. With a ratio $<1.5$, the risk of developing RDS is approximately $73 \% .^{2}$ Therefore many neonates with an immature $\mathrm{L} / \mathrm{S}$ ratio do not develop RDS. Given that this test is more expensive and takes more time to perform, it is currently used only in cases of equivocal results with other tests of lung maturity.

The TDx test is now the most commonly used method to assess fetal lung maturity. The test uses fluorescence polarization to assess surfactant content. The surfactant albumin ratio is determined with amniotic fluid albumin as an internal reference. The newer FLM-II tests use 55 as the mature cutoff. The TDx tests correlate well with the L/S ratio and has few false mature results. Approximately 50\% of infants with immature TDx results develop RDS. ${ }^{2}$ The test has become widely popular as it is automated, can be run in less than an hour, requires only $1 \mathrm{~mL}$ of amniotic fluid, and is of low cost.

The lamellar body count test is also commonly used. Lamellar bodies are the storage form of surfactant released by fetal type II pneumocytes into the amniotic fluid. The amniotic fluid concentration may be determined using a commercial cell counter as lamellar bodies are of the same size as platelets. The test requires $<1 \mathrm{~mL}$ of amniotic fluid and takes only 15 minutes to perform. The test is still reliable; a lamellar body count $>32,000$ predicted a mature $\mathrm{L} / \mathrm{S}$ ratio in $99 \%$ of cases. $^{2}$ 


\section{Indications for Antenatal Testing}

The American Congress of Obstetricians and Gynecologists practice bulletin on antepartum fetal surveillance suggests that antepartum testing may be appropriate for any pregnancy in which there is an increased risk of fetal demise. However, as antepartum fetal surveillance has not been shown to definitively improve perinatal outcome in controlled trials, all indications for antepartum testing must be considered tentative. ${ }^{19}$ Experts currently recommend antenatal testing regimens for many maternal and fetal conditions, such as maternal diabetes, hypertension, and fetal growth restriction (Table 2). ${ }^{19}$ Although observational studies have examined the use of nonstress test and BPP in the management of specific maternal conditions, such as the diabetic pregnancy, no method has been assessed in well-designed clinical trials and it is not clear whether any method is effective and which method, if any, is superior. ${ }^{1}$

\section{Conclusions}

Limited definitive evidence exists regarding the benefit of antenatal testing in decreasing the PMR. Despite this fact, testing is used widely for many different indications. Given this discrepancy, obstetricians should proceed with caution and approach the initiation of a testing protocol by obtaining informed consent from the woman before ordering these tests. Fetal umbilical artery Doppler evaluation should be used for fetal monitoring in high-risk pregnancies thought to be at risk of placental insufficiency. ${ }^{18}$ Other tests should be ordered and interpreted with caution. When confronted with an abnormal fetal test result, physicians should consider the overall clinical picture, taking into account the high possibility that the test result is falsely positive. In the case of acute maternal illness, stabilizing the maternal condition and retesting the fetus may be appropriate. In the scenario where an abnormal test result is not associated with any evidence of worsening maternal status, a sequential approach to further evaluate the fetal condition should be used. ${ }^{19}$ In a preterm pregnancy, the physician should evaluate with a second antenatal test and consider administering betamethasone, performing amniocentesis to assess lung maturity, and/or repeating testing after 24 hours of close observation before making the decision for delivery to minimize the chance of iatrogenic prematurity in the case of a healthy fetus. In postterm pregnancies, the decision to proceed with delivery may be easier with decreased chance of induced morbidity in the case of a false-positive result. Overall, physicians need to proceed with caution before initiating testing given unknown benefit and possibility of harm from unnecessary interventions.

\section{References}

1. Signore C, Freeman RK, Spong CY. Antenatal testing-a reevaluation: executive summary of a Eunice Kennedy Shriver National Institute of Child Health and Human Development workshop. Obstet Gynecol. 2009; 113:687-701. [PubMed: 19300336]

2. Gabbe, SG.; Niebyl, JR.; Simpson, JL. Obstetrics: Normal and Problem Pregnancies. Churchill Livingstone/Elsevier; Philadelphia, PA: 2007.

3. Haws RA, Yakoob MY, Soomro T, et al. Reducing stillbirths: screening and monitoring during pregnancy and labour. BMC Pregnancy Childbirth. 2009; 9(suppl 1):S5, 1-48. [PubMed: 19426468]

4. Winje BA, Saastad E, Gunnes N, et al. Analysis of "count-to-ten" fetal movement charts: a prospective cohort study. BJOG. 2011; 118:1229-1238. [PubMed: 21585644]

5. Moore TR, Piacquadio K. A prospective evaluation of fetal movement screening to reduce the incidence of antepartum fetal death. Am J Obstet Gynecol. 1989; 160(5 Pt 1):1075-1080. [PubMed: 2729383] 
6. Mangesi L, Hofmeyr GJ. Fetal movement counting for assessment of fetal wellbeing. Cochrane Database Syst Rev. 2007:CD004909. [PubMed: 17253530]

7. Grant A, Elbourne D, Valentin L, et al. Routine formal fetal movement counting and risk of antepartum late death in normally formed singletons. Lancet. 1989; 2:345-349. [PubMed: 2569550]

8. Grivell RM, Alfirevic Z, Gyte GM, et al. Ante-natal cardiotocography for fetal assessment. Cochrane Database Syst Rev. 2010:CD007863. [PubMed: 20091654]

9. Enkin, MA. Guide to Effective Care in Pregnancy and Childbirth. Oxford University Press; Oxford, NY: 2000.

10. Blix E, Sviggum O, Koss KS, et al. Inter-observer variation in assessment of 845 labour admission tests: comparison between midwives and obstetricians in the clinical setting and two experts. BJOG. 2003; 110:1-5. [PubMed: 12504927]

11. Freeman RK, Anderson G, Dorchester W. A prospective multi-institutional study of antepartum fetal heart rate monitoring. I. Risk of peri-natal mortality and morbidity according to antepartum fetal heart rate test results. Am J Obstet Gynecol. 1982; 143:771-777. [PubMed: 7102744]

12. Lalor JG, Fawole B, Alfirevic Z, et al. Biophysical profile for fetal assessment in high risk pregnancies. Cochrane Database Syst Rev. 2008:CD000038. [PubMed: 18253968]

13. Manning FA, Morrison I, Harman CR, et al. Fetal assessment based on fetal biophysical profile scoring: experience in 19,221 referred high-risk pregnancies. II. An analysis of false-negative fetal deaths. Am J Obstet Gynecol. 1987; 157(4 Pt 1):880-884. [PubMed: 3674161]

14. Miller DA, Rabello YA, Paul RH. The modified biophysical profile: antepartum testing in the 1990s. Am J Obstet Gynecol. 1996; 174:812-817. [PubMed: 8633648]

15. Alfirevic Z, Stampalija T, Gyte GM. Fetal and umbilical Doppler ultrasound in normal pregnancy. Cochrane Database Syst Rev. 2010:CD001450. [PubMed: 20687066]

16. Karsdorp VH, van Vugt JM, van Geijn HP, et al. Clinical significance of absent or reversed end diastolic velocity waveforms in umbilical artery. Lancet. 1994; 344:1664-1668. [PubMed: 7996959]

17. Tyrrell S, Obaid AH, Lilford RJ. Umbilical artery Doppler velocimetry as a predictor of fetal hypoxia and acidosis at birth. Obstet Gynecol. 1989; 74(3 Pt 1):332-337. [PubMed: 2668815]

18. Alfirevic Z, Stampalija T, Gyte GM. Fetal and umbilical Doppler ultrasound in high-risk pregnancies. Cochrane Database Syst Rev. 2010:CD007529. [PubMed: 20091637]

19. ACOG Practice BulletinAntepartum fetal surveil-lance. Number 9, October 1999 (replaces Technical Bulletin Number 188, January 1994). Clinical management guidelines for obstetriciangynecologists. Int J Gynaecol Obstet. 2000; 68:175-185. [PubMed: 10717828] 
TABLE 1

Fetal Movement Counting “Alarms” - Ability to Predict Poor Outcomes

\begin{tabular}{|c|c|c|c|c|}
\hline & Description & Sensitivity $^{\dagger}(\%)$ & Specificity $^{\dagger}(\%)$ & Positive Alarms $(\mathrm{N}=1786$ Women $)$ \\
\hline 2-h alarm & $<10$ movements in $2 \mathrm{~h}$ & 5 & 96 & $70(4 \%)$ \\
\hline 25/35-min alarm & $<10$ movements in 25 and 35 min $^{*}$ & 44 & 60 & $740(41 \%)$ \\
\hline
\end{tabular}


TABLE 2

Possible Indications for Antenatal Testing

\begin{tabular}{l} 
Maternal indications \\
Hypertensive disorders \\
Hyperthyroidism \\
Hemoglobinopathies \\
Cyanotic heart disease \\
Systemic lupus erythematosus \\
Chronic renal disease \\
Diabetes mellitus \\
Antiphospholipid syndrome \\
Pregnancy-related indications \\
Pregnancy-induced hypertension \\
Decreased fetal movement \\
Oligohydramnios \\
Polyhydramnios \\
Intrauterine growth restriction \\
Postterm pregnancy \\
Isoimmunization \\
Previous fetal demise \\
Chrostiple gestation \\
\hline
\end{tabular}

